\title{
Improvement of photostress recovery testing after extracranial-intracranial bypass surgery
}

\author{
BARRETT KATZ AND PHILLIP R WEINSTEIN \\ From the Departments of Ophthalmology and Neuroscience, University of California, San Diego, and \\ Department of Neurosurgery, University of California, San Francisco, USA
}

SUMmARY A patient with carotid occlusive disease experienced episodes of light-induced amaurosis in an eye with changes of venous stasis retinopathy. Though his macula appeared normal on examination, the macular photostress test (MPST) was prolonged. After anastomosis of a superficial temporal artery with the middle cerebral artery his symptoms resolved and his MPST returned to normal. The MPST may serve as a diagnostic stress test for the retinal circulation in cases of carotid disease.

The macular photostress test (MPST) monitors in a semiquantitive fashion the effect of light on the retina. The test depends on a subject's ability to discern the resolution of a photic-induced scotoma. It has been shown to be a valuable adjunct in differentiating subtle maculopathy and subclinical optic neuropathy, and has prognostic value in predicting recovery or progression of macular disease.'

In this test the macula is dazzled with a hand-held penlight, and the time required for subjectively measured visual acuity to return to prestress levels is compared with normal recovery times. Best corrected acuity for distance is determined. With one eye shielded the patient looks directly into a handheld muscle light for 10 seconds as that light is held 3 $\mathrm{cm}$ from the eye. Immediately after the light is removed the patient is asked to read test letters one line larger than his best acuity. Photostress recovery is recorded in seconds, with the end point being the capacity to read any three letters of that predetermined line. The procedure is then repeated for the opposite eye.

We report experience with a patient whose clinical course and test results suggest the use of the MPST in evaluating patients with carotid occlusive disease.

\section{Case report}

A 60-year-old right-handed native-born white male had been followed up for angiographically confirmed

Correspondence to Barrett Katz, MD, Department of Ophthalmology, UCSD Eye Center (M-018), University of California, La Jolla, CA 92093, USA. atherosclerotic coronary vascular disease. He was not hypertensive or diabetic, and was successfully managed on propranolol and nitroglycerin. In November 1982 he noted transient loss of vision in his left eye precipitated by bright light or mild exercise. Episodes occurred daily and were characterised by diminution of central vision with relative preservation of peripheral vision. They were occasionally accompanied by phosphenes and persisted for 3-5 minutes. His visual loss was not accompanied by any other neurological or neuro-ophthalmic symptoms, and it resolved without sequelae.

In December 1982 he suffered more classical amaurosis of the left eye, accompanied by aphasia. He was admitted to hospital and studied angiographically. His aortic arch showed generalised atherosclerotic irregularity and tortuosity, as did the proximal brachiocephalic vessels. There was a large ulcerated plaque in the region of the right common carotid bifurcation associated with haemodynamically significant stenosis of the proximal parts of the right internal and external carotid arteries. A saccular aneurysm was noted in the region of the anterior communicating artery, and slight atherosclerotic irregularity was present in the right carotid siphon. The left internal carotid artery appeared to be completely occluded at its origin. There was reconstitution of the carotid siphon on the left side via collaterals through the ophthalmic artery, which was hypertrophied (Fig. 1). The external carotid artery showed minimal stenosis proximally.

A left internal carotid endarterectomy was performed with removal of thrombus but unsuccessful 
Fig. 1 Left common carotid arteriogram shown in the lateral projection demonstrates occlusion of the proximal internal carotid artery (ICA) with retrograde flow through an enlarged ophthalmic artery filling the intracranial ICA and its branches.



establishment of back flow. A tight stenosis at the take-off of the left external carotid was observed, and a distal common and external carotid endarterectomy was performed.

Two weeks later his right carotid artery was explored. At operation a tight stenosis of both internal and external carotid arteries was identified, and a plaque was removed from the area of bifurcation. The operation was without complication or sequelae.

Postoperatively there was a decrease in frequency of his light-induced amaurosis and total disappearance of his classical amaurosis fugax. Over the ensuing months no symptoms of hemisphere ischaemia developed. When his light-induced amaurosis increased in frequency, he was referred to us for further examination.

When he was first seen in June 1982 he had small blot haemorrhages and microaneurysms in the midperiphery of his left eye only, accompanied by darkening and mild dilatation of the venous retinal vasculature. The results of macular examination were normal in both eyes (Figs. 2, 3). Fundus fluorescein angiography showed punctate areas of capillary dilatation associated with dot and blot haemorrhages and microaneurysmal formation in the midperiphery of the left eye (Fig. 4). The right eye was ophthalmoscopically and angiographically normal. Ophthalmodynamometry revealed a diastolic reading of 30 scale units on the right and less than 5 scale units on the left. Because of the history of light-induced amaurosis, photostress testing was performed according to the technique of Glaser et al. ${ }^{\prime}$ The right macula recovered function in 10 seconds while the left macula required 80 seconds for recovery. Cerebral blood flow (CBF) measurement after intravenous administration of Xenon-133 showed a blood flow of $36 \mathrm{ml} / \mathrm{min} / 100 \mathrm{~g}$ in the left hemisphere and flow of $51 \mathrm{ml} / \mathrm{min} / 100 \mathrm{~g}$ in the right hemisphere.

To decrease the retinal ischaemia and to increase flow to the hemisphere an anastomosis was performed between a left superficial temporal artery and the middle cerebral artery (STA-MCA). Post- 




Fig. 2 Fundus photograph of posterior pole of the right eye showing normal anatomy.

operatively the patient was entirely free of transient visual loss. Repeat neuro-ophthalmic examination 10 days after surgery revealed no change in the appearance of his left retina. The MPST, however, returned to normal, with a recovery time of less than 10 seconds in that eye. Ophthalmodynamometry still

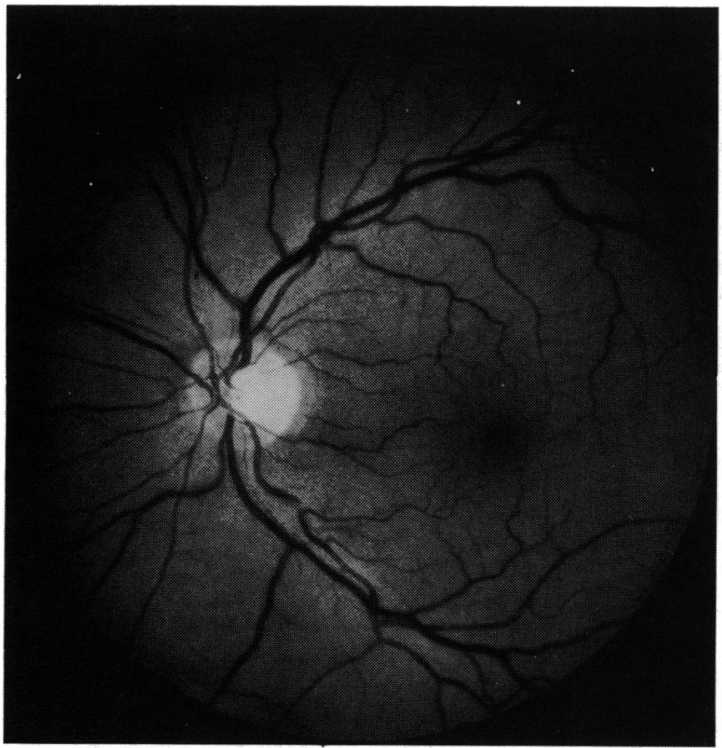

Fig. 3 Fundus photograph of posterior pole of the left eye illustrates darkening and mild dilatation of the venous vasculature. suggested decreased flow in the left central retinal artery.

Four months later the patient remained free of visual symptoms. He underwent follow-up intravenous digital angiography, which revealed patency of the STA-MCA anastomosis. His retinal examination was unchanged, and his MPST remained normal bilaterally.

\section{Discussion}

Venous stasis retinopathy describes the chronic retinal changes of ischaemia due to haemodynamically significant reduction of flow through the internal carotid artery. ${ }^{2}$ It is characterised by small retinal haemorrhages, microaneurysms, and focal areas of capillary dilatation. It includes dilatation, darkening, and tortuosity of the venous side of the retinal circulation and has an anatomical predilection for the midperiphery. The diagnosis is made in the setting of severe chronic occlusive carotid disease, and recognised by the finding of abnormally low central retinal artery pressure on ophthalmodynamometry. Resolution of this retinopathy has been reported after endarterectomy ${ }^{3}$ and after STA-MCA bypass surgery. ${ }^{4.7}$

In 1979 Furlan et al. ${ }^{8}$ described five patients with carotid artery occlusive disease and monocular transient visual loss precipitated by bright sunlight. These patients showed angiographic evidence of highgraded stenosis or occlusion of the ipsilateral carotid

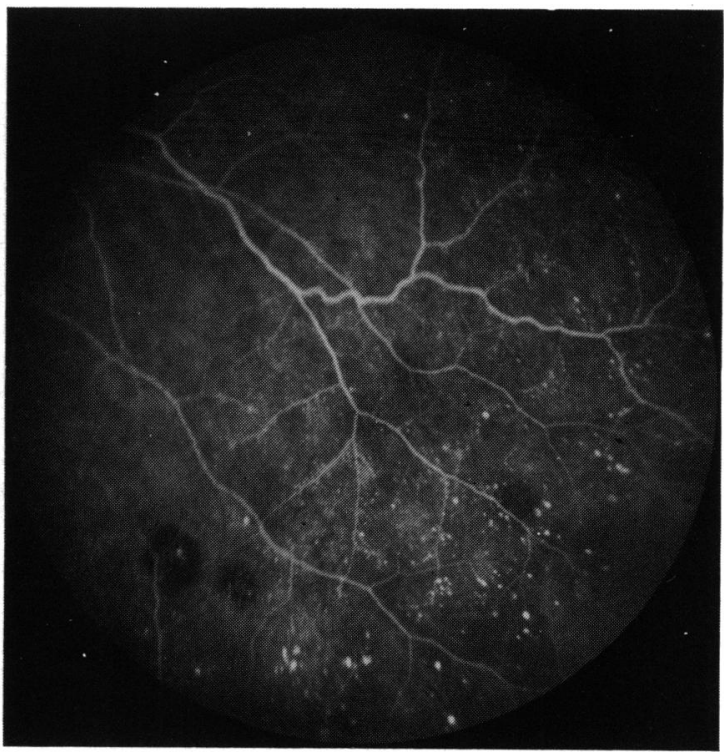

Fig. 4 Fluorescein angiogram of the left fundus illustrates blot haemorrhages and microaneurysms in the midperiphery. 
artery. The authors concluded that such visual loss in patients with severe carotid occlusive disease occurs on the basis of local haemodynamic insufficiency rather than emboli. A recent report affords objective documentation of such light-induced amaurosis by recording an attenuated visually evoked response in the two-minute interval following exposure to light. ${ }^{9}$

Our patient had light-induced amaurosis associated with mild venous stasis retinopathy. He had angiographically confirmed ipsilateral internal carotid artery occlusion, and his left hemisphere was perfused in a retrograde fashion through his ophthalmic artery. Preoperative examination revealed a lower retinal artery pressure as determined by ophthalmodynamometry and prolonged photostress recovery time. He underwent successful STA-MCA bypass surgery, which was followed by the disappearance of his light-induced monocular blackouts and the return of his photostress test results to normal. These changes occurred without any discernible regression of his venous stasis retinopathy or change in retinal artery pressure, suggesting the MPST may be a sensitive and early sign of improved retinal perfusion.

The MPST is primarily a test of macular function, ${ }^{1111}$ if only because of the parameter we use to measure it-macular vision. Recovery from photostress is presumed to be a function of the rate of visual pigment bleaching and resynthesis and dependent on the relation between the retinal pigment epithelium, Mueller cells, and photoreceptors (that is, outer retinal function). We postulate that our patient's photic-induced amaurosis and prolonged photostress test were a manifestation of outer retinal ischaemia secondary to carotid insufficiency. Another possibility is a light-induced photoregulatory change in the inner retinal circulation. In light, more choroidal oxygen diffuses into the inner retina than in the dark. The autoregulatory response of the retinal vasculature to increased local tissue oxygen results in decreased blood flow in the light. ${ }^{12}$ Perhaps in the ischaemic eye the light-induced increased inner retinal oxygen from the choroid drives the vasculature toward vasoconstriction. In either case exposure to light caused further reduction of blood flow to a borderline compensated inner and outer retinal circulation, precipitating light-induced amaurosis.

Abnormal photostress testing has been commonly recognised in macular degeneration, central serous choroidopathy, and disease of the retinal pigment epithelium. We believe the MPST may in selected cases serve as a diagnostic stress test for the autoregulatory capacity of the eye. Deviation from the norm may be a sign of underlying carotid insufficiency.

The improvement of the photostress test results after STA-MCA anastomosis parallels previous reports of the resolution of venous stasis retinopathy and elimination of light-induced amaurosis following extracranial bypass surgery. Although the anastomosis is placed on a cortical branch of the middle cerebral artery at some distance from the carotid origin of the ophthalmic artery, it is postulated that augmentation of collateral flow to the hemisphere through the bypass reduces the 'steal' from the ophthalmic circulation and results in improved blood flow to the eye.

The authors gratefully acknowledge the thoughtful reviews and suggestions of Drs William F Hoyt and John J Weiter.

\section{References}

1 Glaser JS, Savino PJ, Sumers KD, McDonald SA, Knighton $\mathrm{RW}$. The photostress recovery test in the clinical assessment of visual function. Am J Ophthalmol 1977; 83: 255-60.

2 Kearns TP, Hollenhorst RW. Venous stasis retinopathy of occlusive disease in the carotid artery. Mayo Clin Proc 1963; 38: 304-12.

3 Neupert JR, Brubaker RF, Kearns TP, Sundt TM. Rapid resolution of venous stasis retinopathy after carotid endarterectomy. Am J Ophthalmol 1976; 81: 600-2.

4 Kearns TP, Young BR, Piepgras DG. Resolution of venous stasis retinopathy after carotid artery bypass surgery. Mayo Clin Proc 1980; 55: 342-6.

5 Shaw HE, Holmes MW, Fleischer AS. Regression of ischemic oculopathy after carotid artery bypass surgery. In: Smith JL, ed. Neuro-ophthalmology focus. New York: Masson, 1982: 171-5.

6 Ausman JI, Lindsay W, Ramsay RC, Chou SC. Ipsilateral subclavian to external carotid and STA-MCA bypass for retinal ischemia. Surg Neurol 1978; 9: 5-8.

7 Edwards MS, Chater NL, Stanley J. Reversal of chronic ocular ischemia by extracranial-intracranial arterial bypass: case report. Neurosurgery 1980; 7: 480-3.

8 Furlan AJ, Whisnant JP, Kearns TP. Unilateral visual loss in bright light: an unusual symptom of carotid artery occlusive disease. Arch Neurol 1979; 36: 675-6.

9 Dunnan GA, Sharbrough FW, Whisnant JP. Carotid occlusive disease: effect of bright light on visual evoked response. Arch Neurol 1982; 39: 687-9.

10 Severin SL, Tour RL, Kershaw RH. Macular function and the photostress test 1. Arch Ophthalmol 1967; 77: 2-7.

11 Severin SL, Tour RL, Kershaw RH. Macular function and the photostress test 2. Arch Ophthalmol 1967; 77: 163-7.

12 Feke GT, Zukerman R, Green GJ, Weiter JJ. Response of human retinal blood flow to light and dark. Invest Ophthalmol Vis Sci 1983; 24: 136-41.

Accepted for publication 1 August 1985. 